\title{
Anomalous Roughening of Viscous Fluid Fronts in Spontaneous Imbibition
}

\author{
J. Soriano, ${ }^{1}$ A. Mercier, ${ }^{2}$ R. Planet, ${ }^{3}$ A. Hernández-Machado, ${ }^{3}$ M. A. Rodríguez, ${ }^{4}$ and J. Ortín ${ }^{3, *}$ \\ ${ }^{1}$ Experimentalphysik I, Universität Bayreuth, Universitätstraße 30, D-95447 Bayreuth, Germany \\ ${ }^{2}$ École Normale Supérieure, Allée d'Italie 46. F-69364 Lyon, France \\ ${ }^{3}$ Departament d'Estructura i Constituents de la Matèria, Universitat de Barcelona, Av. Diagonal 647, E-08028 Barcelona, Spain \\ ${ }^{4}$ Instituto de Física de Cantabria (IFCA), CSIC-UC, E-39005 Santander, Spain
}

(Received 13 December 2004; published 30 August 2005)

\begin{abstract}
We report experiments on spontaneous imbibition of a viscous fluid by a model porous medium in the absence of gravity. The average position of the interface satisfies Washburn's law. Scaling of the interface fluctuations suggests a dynamic exponent $z \simeq 3$, indicative of global dynamics driven by capillary forces. The complete set of exponents clearly shows that interfaces are not self-affine, exhibiting distinct local and global scaling, both for time $\left(\beta=0.64 \pm 0.02, \beta^{*}=0.33 \pm 0.03\right)$ and space $(\alpha=1.94 \pm 0.20$, $\left.\alpha_{\text {loc }}=0.94 \pm 0.10\right)$. These values are compatible with an intrinsic anomalous scaling scenario.
\end{abstract}

DOI: 10.1103/PhysRevLett.95.104501

PACS numbers: 47.55.Mh, 05.40.- a, 68.35.Ct

The dynamics of immiscible fluid-fluid displacements in porous media has been a subject of much interest in last years [1-13], both from a fundamental point of view, as a dynamical nonequilibrium process leading to rough interfaces [1], and from a technological point of view, in industrial and environmental problems such as oil recovery, irrigation, and filtration [2]. The process is called spontaneous imbibition when an invading fluid that wets preferentially the medium displaces a resident fluid at constant external pressure. In contrast, forced-flow imbibition takes place when the invading fluid gets into the porous medium at a constant injection rate.

The case of spontaneous imbibition has special interest from the point of view of dynamic scaling. Spontaneous imbibition is dominated by capillarity. In contrast to forced-flow imbibition, there is no global mass conservation law, a fact that may have a dramatic effect on scaling $[10,11]$. Spontaneous imbibition is a case with slowingdown dynamics in which time scales change continuously. Indeed, it has been known from a long time [14] that the temporal scaling of the mean advancing front obeys Washburn's law, $\langle h\rangle \sim t^{1 / 2}$, but little is known about the scaling of fluctuations in this regime. Moreover, most experiments in spontaneous imbibition have been performed with the disordered medium placed vertically [9], a situation where gravity limits the Washburn behavior to a short observation time. Experiments with gravity have been directed to the study of the pinning process and its scaling. Only one experiment with the disordered medium placed horizontally has been performed on paper [4]. In this experiment, scale invariant roughness is found only for highly anisotropic disorder, while logarithmic roughness, at best, is found in isotropic paper. Paper, however, is a medium with uncontrolled disorder, and phenomena such as swelling and prewetting might change the effect of static capillary forces.

In this Letter we report on spontaneous imbibition experiments of a viscous fluid by a model porous medium with well controlled disorder, consisting on a horizontal Hele-Shaw cell with random variations in gap spacing. Our scaling results suggest a dynamic exponent $z \simeq 3$, consistent with a nonlocal dynamics driven by capillary forces. This exponent has never been observed in experiments before, despite having been suggested by simple models of imbibition $[1,12]$. After a detailed scaling analysis of the interfacial fluctuations we find different global and local exponents, with values that suggest that our experiment must be described in the framework of intrinsic anomalous scaling [15].

Experimental setup. - The experimental setup [6,7] consists on a Hele-Shaw cell of size $190 \times 500 \mathrm{~mm}^{2}(L \times H)$ made of two glass plates separated by a narrow gap of thickness $b=0.46 \mathrm{~mm}$. Fluctuations in the gap spacing

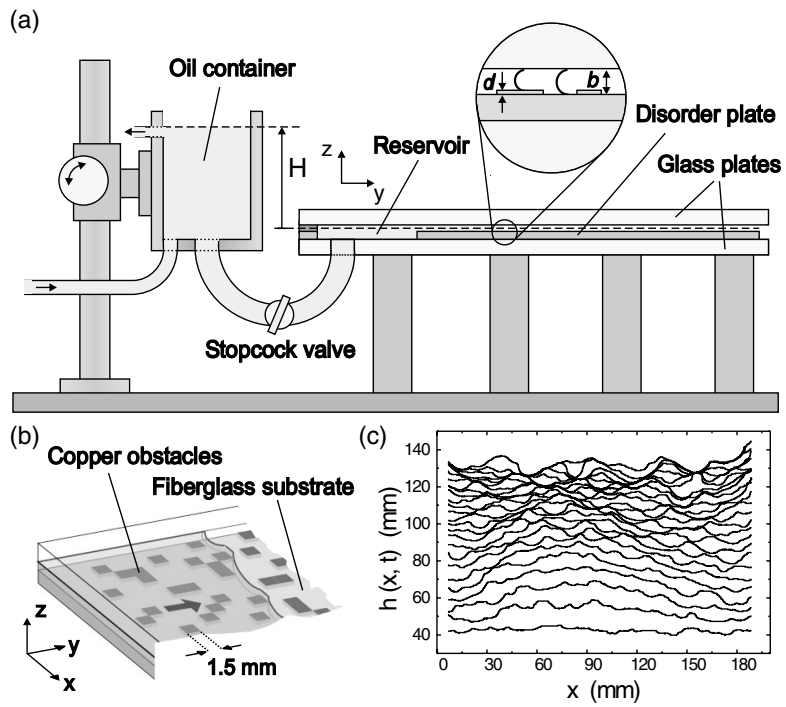

FIG. 1. (a) Sketch of the experimental setup. (b) Schematic representation of our Hele-Shaw cell with quenched disorder in the bottom plate. (c) Temporal evolution of the oil-air interface at $H=-9 \mathrm{~mm}$. 
are provided by square (SQ) copper obstacles $(d=$ $0.06 \mathrm{~mm}$ high, $1.50 \times 1.50 \mathrm{~mm}^{2}$ size) that are randomly distributed over a fiberglass substrate, without overlap, filling 35\% of the substrate area (disorder SQ 1.50 in Ref. [6]).

In the present set of experiments we use a constant pressure device that consists on an oil container of selectable height $H$, in the range from -50 to $100 \mathrm{~mm}$, in steps of $0.1 \mathrm{~mm}$. A silicone oil (Rhodorsil $47 \mathrm{~V}$ ) with kinematic viscosity $\nu=50 \mathrm{~mm}^{2} / \mathrm{s}$, density $\rho=998 \mathrm{~kg} / \mathrm{m}^{3}$, and oilair surface tension $\sigma=20.7 \mathrm{mN} / \mathrm{m}$ at room temperature, penetrates into the cell through two wide holes drilled in the bottom glass, displacing the air initially present. The holes connect to a reservoir in the cell, with the disorder plate placed just ahead (Fig. 1).

The experimental procedure used to prepare a flat initial interface is the following: The reservoir in the cell is carefully filled and, using an auxiliary syringe, the oil-air interface is pushed gently up to a transverse copper track $2 \mathrm{~mm}$ behind the disorder pattern, and the experiment starts. The evolution of the oil-air interface is monitored using two CCD cameras. A computer records the images acquired and stores them for processing.

Washburn's law. - Different heights of the oil column in the container, in the range $-9 \leq H \leq 15 \mathrm{~mm}$, have been explored. One example of the temporal evolution of the interface is shown in Fig. 1(c) for $H=-9 \mathrm{~mm}$. As a first result (Fig. 2) we observe that for $H>-10 \mathrm{~mm}$ the average interface position obeys Washburn's law, $\langle h\rangle=A t^{1 / 2}$. For $H<-10 \mathrm{~mm}$ the interfaces recede. By Darcy's law $\langle\dot{h}\rangle \sim \nabla p \sim H /\langle h\rangle$, so that $A^{2} \sim H$, as verified in the inset of Fig. 2.

Scaling analysis of the rough fronts. - The statistical properties of a one-dimensional interface defined by a function $h(x, t)$ are usually described in terms of the fluctuations of $h$. More precisely, global fluctuations are measured by the global interface width, which for a system of

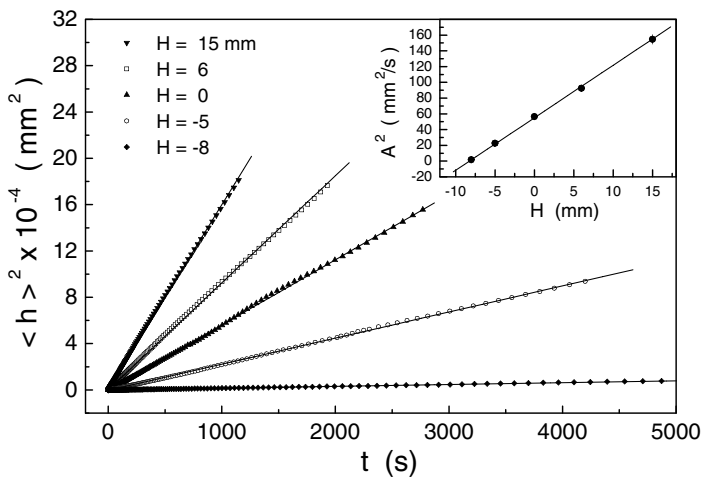

FIG. 2. Main plot: Squared average interface position as a function of time, for different oil column heights $H$, showing that the imbibition process follows Washburn's law, $\langle h\rangle=A t^{1 / 2}$. Inset: Dependence of Washburn's law prefactor $A$ (squared) on column height $H$. total lateral size $L$ scales according to the Family-Vicsek (FV) ansatz [16]. However, in experiments one cannot usually probe the global width, since system size is fixed in standard setups. Instead of global fluctuations, one measures the local surface fluctuations, which are more easily accessible in the laboratory. Local interface fluctuations are measured by calculating either the spectral density of the interface, $S(k, t) \equiv\langle h(k, t) h(-k, t)\rangle$, where $h(k, t)$ is the Fourier transform of the interface and brackets indicate averages over independent runs of the experiment, or the local width, $w(l, t)=\left\langle\left\langle\left[h(x, t)-\langle h\rangle_{l}\right]^{2}\right\rangle_{l}\right\rangle^{1 / 2}$, where $\langle\cdots\rangle_{l}$ denotes an average over $x$ in windows of size $l$. The local width is also expected to satisfy dynamic scaling

$$
w(l, t)=t^{\beta} g\left(l / t^{1 / z}\right),
$$

where the most general scaling function $[15,17]$ is expected to behave as

$$
g(u) \sim\left\{\begin{array}{ll}
u^{\alpha_{\mathrm{loc}}} & \text { if } u \ll 1 \\
\text { const. } & \text { if } u \gg 1
\end{array} .\right.
$$

Here $\beta$ and $z$ are the growth and dynamic exponents, respectively, and $\alpha=\beta z$ is the roughness exponent. So, for short scales $\left(l<t^{1 / z}\right)$ the local width grows as $t^{\beta^{*}}$ with $\beta^{*}=\left(\alpha-\alpha_{\text {loc }}\right) / z$, whereas for larger scales the growth of the global width, $t^{\beta}$, is recovered. This generalization of the scaling form is required to account for potentially anomalous roughening $\left(\alpha \neq \alpha_{\text {loc }}\right)$. Note that three independent exponents are necessary now to define the universality class. The standard self-affine FV scaling [16] is then fully recovered when global and local roughness exponents become equal, i.e., $\alpha=\alpha_{\text {loc }}, \beta^{*}=0$. Sometimes a more detailed analysis of the scaling functions is necessary to completely define the generic class of scaling. Then both $S(k, t)$ and $w(l, t)$ must be examined. The scaling of the spectrum

$$
S(k, t)=k^{-(2 \alpha+1)} s\left(k t^{1 / z}\right),
$$

where now the scaling function is

$$
s(u) \sim\left\{\begin{array}{ll}
u^{2\left(\alpha-\alpha_{s}\right)} & \text { if } u \gg 1 \\
u^{2 \alpha+1} & \text { if } u \ll 1
\end{array},\right.
$$

and $\alpha_{s}$ is the spectral exponent, adds valuable additional information [15]. With the same value of the scaling exponents the system can belong to one or another class depending on the value of the auxiliary exponent $\alpha_{s}$. For instance, with $\alpha>1$ the system can be either in the superrough, $\alpha_{s}=\alpha$, or the intrinsic anomalous class, $\alpha_{s}=$ $\alpha_{\mathrm{loc}}$. This is important because it can inform of the existence of different symmetries [18].

Experimental results. - Since slight perturbations of the interface at the beginning of the experiment are unavoidable, it is difficult to characterize the scaling of the interfacial fluctuations at very short times. To minimize this effect we have always considered the subtracted width $W(l, t)$, defined as $W(l, t)=\left[w^{2}(l, t)-w^{2}(l, 0)\right]^{1 / 2}[3]$. 
We have focused on measuring the values of the scaling exponents for an oil column height $H=-9 \mathrm{~mm}$. In these conditions the imposed (negative) pressure gradient nearly compensates the traction due to capillarity, so that the interface is driven very slowly. The prefactor of Washburn's law in this case is $A=1.26 \pm 0.15 \mathrm{~mm} / \mathrm{s}^{1 / 2}$, and we observe that the average interface velocity (initially $\langle\dot{h}(t=0)\rangle \simeq 0.05 \mathrm{~mm} / \mathrm{s})$ decreases by about $84 \%$ in the experiment. We have performed a total of 6 different experiments, 2 for each of 3 different disorder realizations. The plot of $W(l, t)$ as a function of time and for 9 different windows of size $l$ is shown in Fig. 3(a). We obtain a power law with different exponents for large and small window size, $\beta=0.64 \pm 0.02$ and $\beta^{*}=0.33 \pm 0.03$. The value of this last exponent is very robust and clearly different from 0 . Saturation occurs simultaneously in all scales at around $6800 \mathrm{~s}$. Both facts rule out a FV scaling and indicate the presence of anomalous scaling.

The exponent $\alpha_{\text {loc }}$ can be obtained directly from $W(l, t)$ as a function of window size $l$ near saturation. As shown in Figure 3(b) the behavior is close to the expected power law,
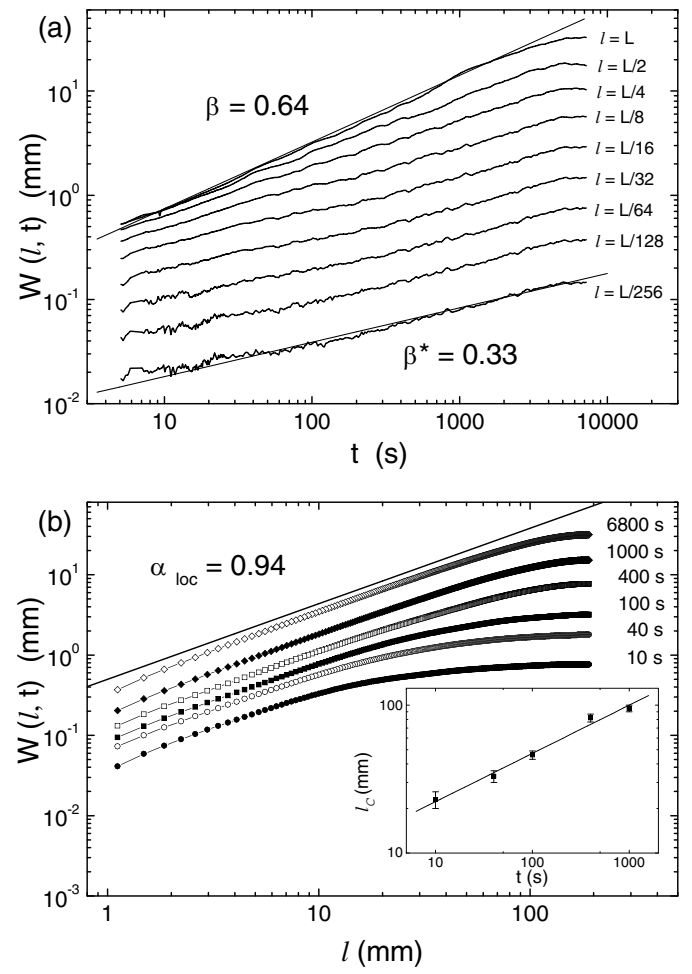

FIG. 3. (a) Log-log plot of the interfacial width as a function of time, for different window sizes $l$. The straight lines, with slopes 0.64 and 0.33 , result from a data fit in the scaling region. (b) Log$\log$ plot of the interfacial width as a function of window size $l$ for different times. The straight line is a fit to the data at the latest time available, with slope 0.94 . The inset shows the correlation length $l_{c}$ as a function of time. The behavior $t^{1 / 3}$ is represented by the solid line. The experimental parameters are $b=0.46 \mathrm{~mm}$ and $H=-9 \mathrm{~mm}$. with an exponent $\alpha_{\mathrm{loc}}=0.94 \pm 0.10$. Having determined the three independent exponents $\beta, \beta^{*}$, and $\alpha_{\mathrm{loc}}$ directly from the experimental data, the remaining exponents $\alpha=$ $1.94 \pm 0.20$ and $z=3.0 \pm 0.3$ follow from the scaling relations.

Figure 3(b) shows also $W(l, t)$ at different time intervals, providing a direct observation of the temporal growth of a lateral correlation length, $l_{c} \sim t^{1 / z}$, as implied by Eqs. (1) and (2). We have estimated $l_{c}$ from the crossover between the power law and plateau regions, and plotted it as a function of time in the inset. The uncertainty in the values of $l_{c}$ determined in this way is large, but the result is consistent with $l_{c} \sim t^{1 / 3}$, as expected.

The exponents obtained provide a good collapse of our data, as presented in Fig. 4(a), consistently with the scaling assumption of Eqs. (1) and (2). Finally, the power spectrum at different times, presented in Fig. 4(b), provides the value $\alpha_{s}=0.92 \pm 0.11$, close to $\alpha_{\text {loc }}$ and significantly different from $\alpha$. This, together with the observation that the spectra at different times are shifted upwards, points unambiguously to an intrinsic anomalous scaling. We have verified that the collapse of the spectrum (not shown in the figure) is also well fitted with the exponents obtained.
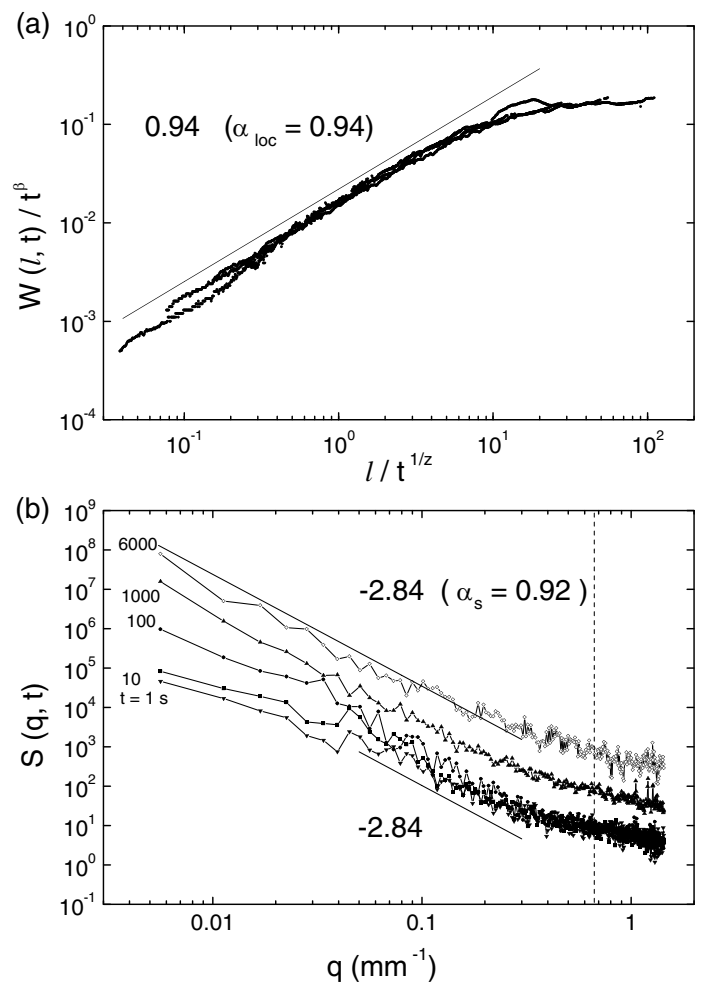

FIG. 4. (a) Collapse of the interfacial width of Fig. 3, using the values $\beta=0.64$ and $z=3.0$. The straight line is the result from a data fit. (b) Power spectra of the interfaces analyzed in Fig. 3 at different times. The variable $q=k /(2 \pi)$. The solid straight lines, with slope -2.84 , are fits to the data in the scaling regions. The vertical dashed line indicates the value $q=0.67 \mathrm{~mm}^{-1}$ associated with the lateral size of the unit copper obstacle. 
Discussion. - The deterministic linearized equation for the dynamics of a perturbation of the interface, in the form of a normal mode of wave number $k$, in spontaneous imbibition, reads $[10,12] \dot{h}_{k}=-\sigma\left(b^{2} / 12 \mu\right) k^{2}|k| h_{k}-$ $\langle\dot{h}\rangle|k| h_{k}$, where $\mu=\rho \nu$ is the dynamic viscosity of the fluid, and $\langle\dot{h}\rangle$ is the average interface velocity. Surface tension (the term proportional to $k^{2}|k|$ ) is responsible for the damping of short wavelength fluctuations, while viscous pressure (the term proportional to $\langle\dot{h}\rangle|k|$ ) damps long wavelength fluctuations.

The two terms, however, cross over at a characteristic length $\xi_{\times}=2 \pi\left[\sigma\left(b^{2} / 12 \mu\right)(1 /\langle\dot{h}\rangle)\right]^{1 / 2}$. In spontaneous imbibition, $\langle\dot{h}\rangle \sim t^{-1 / 2}$, and thus the crossover length $\xi_{\times} \sim t^{1 / 4}$. As the interface invades the porous medium, Dubé et al. [10] have pointed out that the growth of the lateral correlation length due to the $k^{2}|k|$ term, $l_{c} \sim t^{1 / 3}$, is effectively dominated by the slower growth of the crossover length, $\xi_{\times} \sim t^{1 / 4}$. One should then observe a dynamic exponent $z=4$ for length scales larger than $\xi_{\times}$, a result verified in Ref. [9].

In our experiment, however, $l_{c}$ never reaches $\xi_{\times}$, and thus the observation of a dynamic exponent $z \simeq 3$ is fully justified on theoretical grounds. Indeed, (i) at very short times $l_{c}$ grows from 0 while, from $\langle\dot{h}(t=0)\rangle \simeq 0.05 \mathrm{~mm} / \mathrm{s}$ and the values of $\sigma, b$, and $\mu$, we get $\xi_{\times} \simeq 76 \mathrm{~mm}$, which is about half the cell width. (ii) At saturation $\left(l_{c}=L\right) \xi_{\times}$ can be estimated as follows: $l_{c}=(D t)^{1 / 3}$, where $D \simeq$ $10^{3} \mathrm{~mm}^{3} / \mathrm{s}$ is estimated from the inflection in Fig. 4(a), so that saturation occurs indeed at a time $t_{s}=L^{3} / D \simeq$ $6800 \mathrm{~s}$. The interface velocity at $t_{s}$, determined experimentally, is $8 \times 10^{-3} \mathrm{~mm} / \mathrm{s}$. From this velocity we get $\xi_{\times} \simeq$ $190 \mathrm{~mm}$ at saturation; a value that coincides with the size of the cell. Thus $\xi_{\times}$is only reached at saturation. (iii) This result is consistent with the observation that at the end of the experiment $\langle\dot{h}\rangle \simeq 0.16\langle\dot{h}(t=0)\rangle$, and we get again $\xi_{\times} \simeq 190 \mathrm{~mm}$. This reflects also that saturation is reached shortly before the end of the experiment.

Conclusions. - Our experiments provide a controlled realization of spontaneous imbibition without gravity. The interface roughness is a result of the random spatial distribution of capillary forces. Traction due to capillarity is so strong that the external pressure must be negative to have a very slow (forward) motion of the roughening front. Once the control parameter (the pressure imposed) is fixed, the front advance verifies Washburn's law.

Our results $\alpha \simeq 2, \alpha_{\mathrm{loc}} \simeq 1, \beta \simeq 2 / 3, \beta^{*} \simeq 1 / 3$, and $z \simeq$ 3 , together with $\alpha_{s} \simeq \alpha_{\text {loc }}$, identify the present experiment with the so-called intrinsic anomalous scaling [15]. This type of scaling is entirely different from the usual FV scaling found in the same setup and with the same kind of disorder, in forced-flow conditions [6]. This substantial difference should be attributed to the absence of a global mass conservation law.

Finally, our results provide the first experimental evidence of $z \simeq 3$ in spontaneous imbibition. This has been made possible by driving the interfaces very slowly, thus avoiding the lateral correlation length $l_{c}$ to reach the crossover length $\xi_{\times}$in the experiment. Preliminary experiments in which the interfaces are driven at much higher pressure $(H=29 \mathrm{~mm})$ and, consequently, $\xi_{\times}$is much smaller throughout the whole experiment, cannot be properly collapsed with $z=3$.

We acknowledge fruitful discussions with J. M. López and M. Pradas and the technical support of A. Comerma and M. Quevedo. J.S. acknowledges the financial support of the European Training Network PHYNECS through Project No. HPRN-CT-2002-00312, and R.P. acknowledges support from the DGI (MEC, Spain). This work is supported through Project Nos. BFM2003-07749-C05, BQU2003-05042-C02-02, and SGR-2000-00433.

*Electronic address: ortin@ecm.ub.es

[1] M. Alava, M. Dubé, and M. Rost, Adv. Phys. 53, 83 (2004).

[2] M. Sahimi, Flow and Transport in Porous Media and Fractured Rock (John Wiley \& Sons, New York, 1995).

[3] A.-L. Barabási and H.E. Stanley, Fractal Concepts in Surface Growth (Cambridge University Press, Cambridge, England, 1995).

[4] O. Zik et al., Europhys. Lett. 38, 509 (1997).

[5] M. A. Rubio et al., Phys. Rev. Lett. 63, 1685 (1989); V. K. Horváth, F. Family, and T. Vicsek, J. Phys. A 24, L25 (1991); S. He, G. L. M. K. S. Kahanda, and P.-Z. Wong, Phys. Rev. Lett. 69, 3731 (1992).

[6] J. Soriano, J. Ortín, and A. Hernández-Machado, Phys. Rev. E 66, 031603 (2002).

[7] J. Soriano et al., Phys. Rev. Lett. 89, 026102 (2002).

[8] J. Soriano, J. Ortín, and A. Hernández-Machado, Phys. Rev. E 67, 056308 (2003).

[9] D. Geromichalos, F. Mugele, and S. Herminghaus, Phys. Rev. Lett. 89, 104503 (2002).

[10] M. Dubé et al., Phys. Rev. Lett. 83, 1628 (1999).

[11] M. Dubé et al., Phys. Rev. E 64, 051605 (2001).

[12] A. Hernández-Machado et al., Europhys. Lett. 55, 194 (2001).

[13] E. Pauné and J. Casademunt, Phys. Rev. Lett. 90, 144504 (2003).

[14] E. W. Washburn, Phys. Rev. 17, 273 (1921).

[15] J. J. Ramasco, J. M. López, and M. A. Rodríguez, Phys. Rev. Lett. 84, 2199 (2000).

[16] F. Family and T. Vicsek, J. Phys. A 18, L75 (1985).

[17] J. M. López, M. A. Rodríguez, and R. Cuerno, Phys. Rev. E 56, 3993 (1997).

[18] J. M. López, M. Castro, and R. Gallego, Phys. Rev. Lett. 94, 166103 (2005). 